\title{
Parametric Design and Static Performance Comparison of Six Typical Spherical Semi-open Reticulated Shell
}

\author{
Junchao $\mathrm{Cao}^{1}$, Lili Huang ${ }^{2}$, Xiaoyang $\mathrm{Lu}^{1 *}$, Dayong $\mathrm{Li}^{3}$ and Shuai Chong ${ }^{1}$ \\ ${ }^{1}$ Institute of Engineering Mechanics, Shandong Jianzhu University, Jinan, Shandong, 250001, China \\ ${ }^{2}$ School of Materials, Shandong Jianzhu University, Jinan, Shandong, 250001, China \\ ${ }^{3}$ Dean's Office, Shandong Jianzhu University, Jinan, Shandong, 250001, China
}

\begin{abstract}
By using ANSYS Parametric Design Language (APDL), this paper compiled the parametric design macro program for six types of single-layer semi-open spherical reticulated shell structure. Parametric design of the six types of reticulated shell structure was realized with given parameters of span S, the vector high $\mathrm{F}$, radial node cycles number $\mathrm{Nx}$, ring to symmetric regional copies number $\mathrm{Kn}$, and the upper removed laps Ns. The stress performance of the six types of reticulated shell structure was compared and analyzed. Modelling examples demonstrated that the parametric design macro program is simple, practical and improves the efficiency of the selection of the reticulated shell design and the stress analysis under different geometric parameters. Through the comparative analysis of stress performance, some conclusions with engineering significance were obtained.
\end{abstract}

\section{Introduction}

The semi-open reticulated shell structure is a new curved space mesh structure, which has beautiful shape, reasonable stress, and both the characteristics of the rod system and thin shell structure.It has a broad development space and application prospect [1]. Brazil Bellario Stadium (figure 1), Jieyang Nianci Stadium (figure 2) and Daqing Olympics Stadium (figure 3) are application good examples.

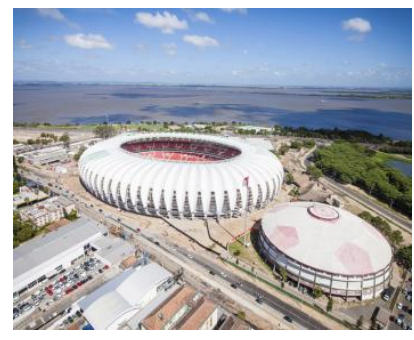

Figure 1. Brazil Bellario Stadium.

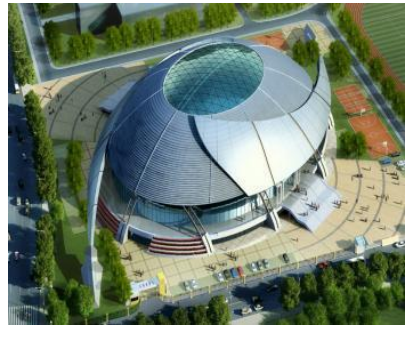

Figure 2. Jieyang Nianci Stadium.

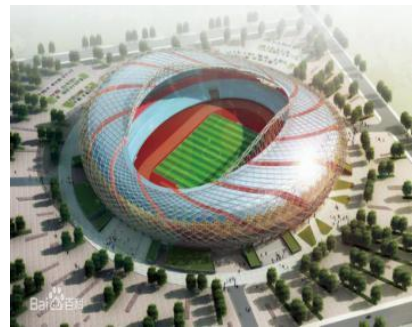

Figure 3. Daqing Olympics Stadium.
The changes of the parameters such as the type, span, vector high, ring to symmetric regional copies number and radial node cycles will result in the redistribution of the internal forces and change of the structure cost [1]. In the stress analysis and optimization of the reticulated shell, the workload of re-modeling is large [2-3]. Therefore, studying the distribution law of rods and nodes of different types of reticulated shell, and applying the parametric design method of the semi-open reticulated shell based on the typical reticulated shell [4], can improve the efficiency of structural design, stress analysis and the selection and optimization design of various semi-open spherical reticulated shell [5-7].

\section{Geometric description of structure}

The main geometric parameters are: span $S$, the vector high $F$, radial node cycles number $N x$, ring to symmetric regional copies number $K n$ and upper removed laps $N s$. The geometric description of ribbed semi-open spherical reticulated shell are shown in figure 4 . 


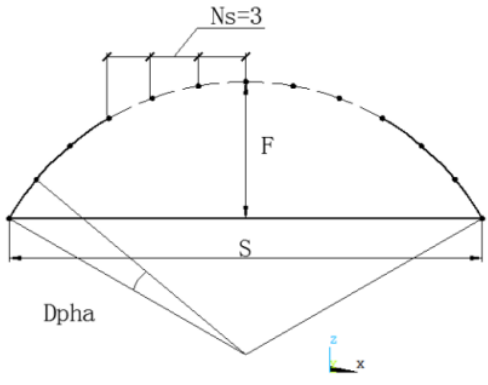

(a) Front view

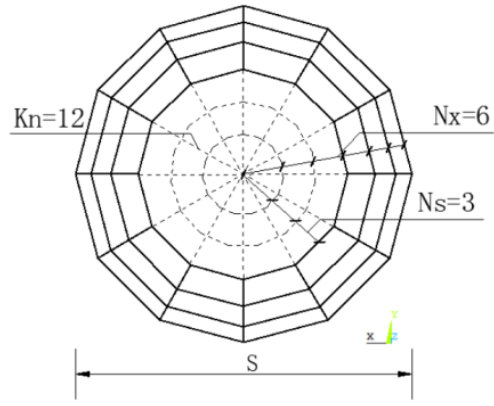

(b) Top view

Figure 4. The main parameters and schematic diagram of ribbed semi-open reticulated shell.

\section{Parametric design of semi-open reticulated shell}

The parametric design method of the Kiewit semi-open reticulated shell is introduced in this section. According to the sports building design code [8], the structure size is selected as: $S=120 \mathrm{~m}, F=45 \mathrm{~m}, K n=6, N x=6, N s=3$.

(1) Determining input control parameters: $\operatorname{span} S$, the vector high $F$, radial node cycles number $N x$, ring to symmetric regional copies number $K n$ and upper removed laps $N s$.

(2) Naming nodes and determining their coordinates. Establishing a spherical coordinate system, the nodes of each circle are named from the $N s$-th circle. Then numbering the nodes at the $i$-th $(N s \leq i \leq N x)$ circle and $j$-th $(1 \leq j \leq K n)$ symmetrical region as $1+k n \times(i-1) \times i / 2+j$. The node coordinates of these nodes are $(R,(j-1) \times 360 /(k n \times i)$, 90-i $\times$ Dpha).

(3) Loop rod connection. The loop rods are connected by the node $1+k n \times(i-1) \times i / 2+j$ and the node $1+k n \times(i-$ $1) \times i / 2+j+1$ at the $i$-th $(N s \leq i \leq N x)$ circle and $j$-th $(1 \leq j \leq k n \times i-1)$ area. The loop rods at the last symmetrical region is connected by the first node and the last node of each circle.

(4) Diagonal rod connection. The left diagonal rods are connected by the node $1+k n \times(j-1) \times j / 2+(k-1) \times j+i$ and $1+k n \times(j+1) \times j / 2+(k-1) \times(j+1)+i$ at the $j$-th $(1 \leq j \leq k n \times i-1)$ region, $i$-th $(N s \leq i \leq N x-1)$ and $i$-th $(N s \leq i \leq N x-1)$ circle. The last left diagonal rod is connected by the node $1+k n \times(j-$ $1) \times j / 2+1$ and $1+k n \times(j+1) \times j / 2+(k-1) \times(j+1)+i$. The right diagonals are connected by the node $1+k n \times(j-1) \times j / 2+(k$ 1) $\times j+i$ and $1+k n \times(j+1) \times j / 2+(k-1) \times(j+1)+i+1$.

(5) Applying node load and boundary constraint. Since the number of nodes increases successively from the inner circle to the outer circle, it can be obtained the first node at last circle is numbered as $1+k n \times(n x-$ $1) \times n x / 2+1$. Applying load when node number is smaller than the first node number. Otherwise, the movable hinge or fixed hinge shall be applied as required by the project.

\section{$4 \quad$ Parametric design examples of six typical spherical semi-open reticulated shell}

Because the Geodesic type and the Three Way Grid type $K n$ can only be 6 or 5 , so the Kiewit type $K n=6$, others $K n=26$ is selected to make the number of joints and rod comparable. The modeling examples of six semi-open spherical reticulated shell are shown in figure 5 when $S=120 \mathrm{~m}, F=45 \mathrm{~m}, N x=6, N s=3$. The total number of structural nodes, circular members, radial members and structural members corresponding to these six kinds of reticulated shell and different $K n$ are shown in table 1.

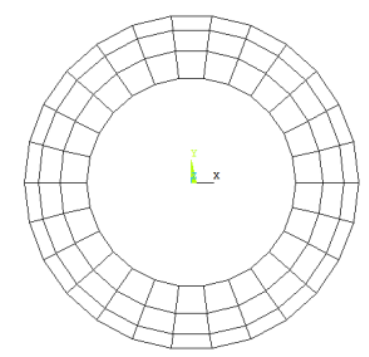

(a) Ribbed semi-open shell $K n=26$

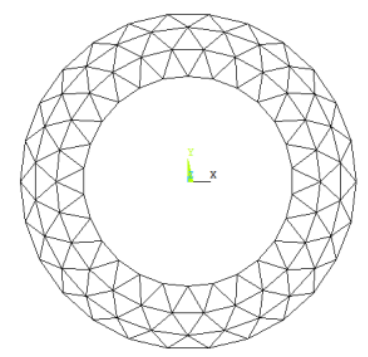

(b) Lamella semi-open shell $K n=26$

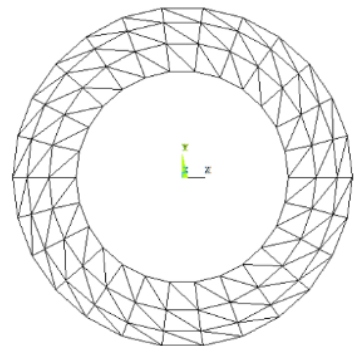

(c) Schwedler semi-open shell $K n=26$ 


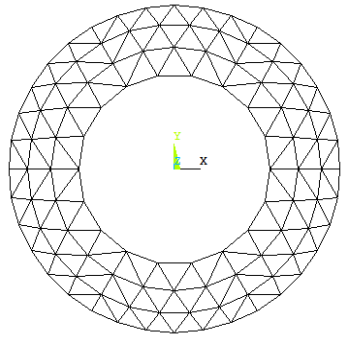

(d) Kiewit semi-open shell $K n=6$

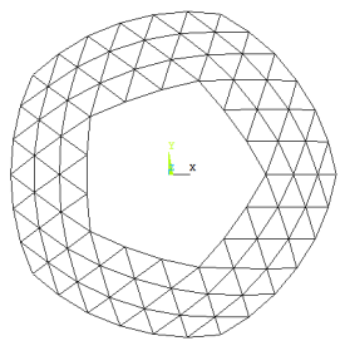

(e) Geodesic semi-open shell $K n=5$

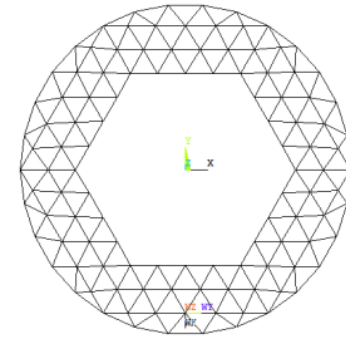

(f) Three Way Grid semi-open shell $K n=6$

Figure 5. Six examples of parametric design of semi-open spherical reticulated shell.

Table 1. Six different types of semi-open reticulated shell parameters and corresponding changes.

\begin{tabular}{|c|c|c|c|c|c|c|c|c|c|c|c|c|}
\hline $\begin{array}{c}\text { Types of } \\
\text { shell }\end{array}$ & $\begin{array}{c}\text { Three } \\
\text { Way Grid }\end{array}$ & Geodesic & Kiewit & \multicolumn{3}{|c|}{ Ribbed } & \multicolumn{3}{|c|}{ Lamella } & \multicolumn{3}{|c|}{ Schwedler } \\
\hline $\mathrm{S}$ & 120 & 120 & 120 & & 120 & & & 120 & & & 120 & \\
\hline $\mathrm{F}$ & 45 & 45 & 45 & & 45 & & & 45 & & & 45 & \\
\hline $\mathrm{Nx}$ & 6 & 6 & 6 & & 6 & & & 6 & & & 6 & \\
\hline Ns & 3 & 3 & 3 & & 3 & & & 3 & & & 3 & \\
\hline $\mathrm{Kn}$ & 6 & 5 & 6 & 25 & 26 & 27 & 25 & 26 & 27 & 25 & 26 & 27 \\
\hline $\begin{array}{l}\text { Structural } \\
\text { nodes }\end{array}$ & 114 & 90 & 108 & 100 & 104 & 108 & 100 & 104 & 108 & 100 & 104 & 108 \\
\hline Loop rod & 120 & 90 & 108 & 100 & 104 & 108 & 100 & 104 & 108 & 100 & 104 & 108 \\
\hline $\begin{array}{l}\text { Diagonal } \\
\text { rod }\end{array}$ & 168 & 135 & 162 & 75 & 78 & 81 & 150 & 156 & 162 & 150 & 156 & 162 \\
\hline $\begin{array}{l}\text { Structural } \\
\text { members }\end{array}$ & 288 & 225 & 270 & 175 & 182 & 189 & 250 & 260 & 270 & 250 & 260 & 270 \\
\hline
\end{tabular}

Note: the shadow data in the table directly affects the weight and cost of the structure with different $K n$.

The following conclusions can be obtained from figure 5 and table 1.

(1) When the reticulated shell covers the same space (that is, $S, F, N x, N s$ are the same), the changes of reticulated shell type and $K n$ will cause the total number of structural nodes and members to change greatly. Moreover, the internal force of the structure will be redistributed, and the stress performance of the reticulated shell structure will also change greatly.

(2) With the increase in the number of nodes and bars, the dead weight and cost of the structure will increase accordingly. The increase and the change of the stress performance of net shell are the factors which should be paid attention to in the selection and optimization design of the reticulated shell.

\section{Stress behavior of semi-open spherical reticulated shell structure}

The structure size is selected as: $S=120 \mathrm{~m}, F=45 \mathrm{~m}, N x=6$, $N s=3$. The form of structural support is fixed hinge support. Uniform load is applied here and the value is $q=2.35 \mathrm{kN} / \mathrm{m}^{2}$ [9]. The dead weight of the structure is considered. The structural members are Q235 steel tubes with a section area of $46.62 \mathrm{~cm}^{2}(\mathrm{~d}=219 \mathrm{~mm}, \mathrm{t}=7 \mathrm{~mm})$. The maximum allowable displacement of the structure is $1 / 400$ of the span [10]. The allowable stress is $215 \mathrm{Mpa}$. Figure 6 and figure 7 show the displacement and stress cloud diagrams. Table 2 and table 3 show the calculated and analyzed data.

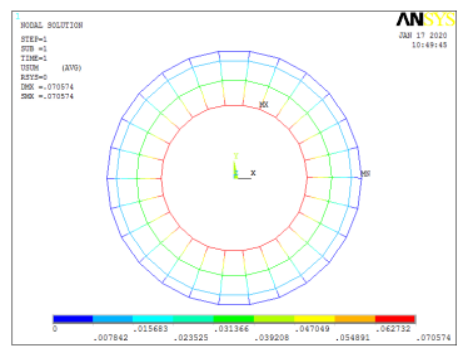

(a) Ribbed semi-open shell $K n=26$

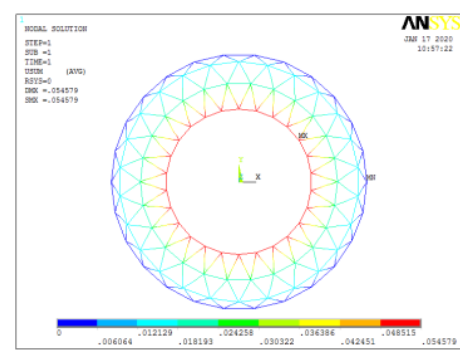

(b) Lamella semi-open shell $K n=26$

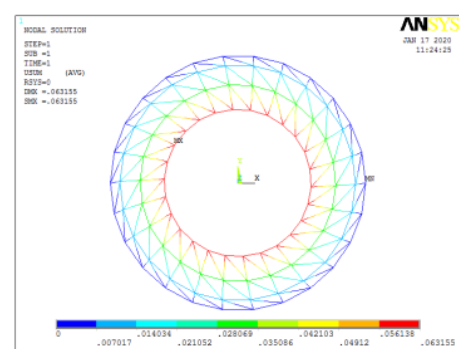

(c) Schwedler semi-open shell $K n=26$ 


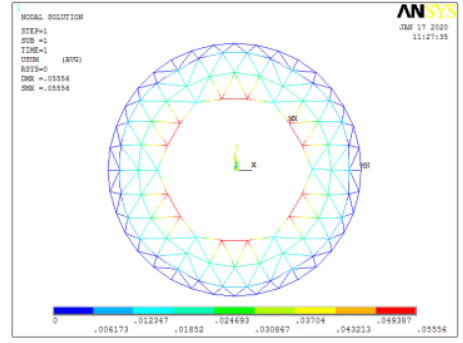

(d) Kiewit semi-open shell $K n=6$

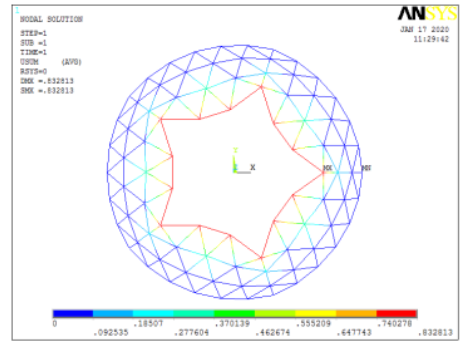

(e) Geodesic semi-open shell $K n=5$

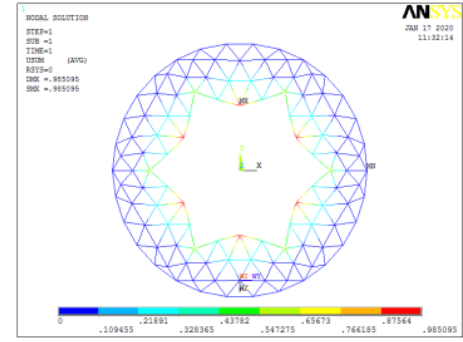

(f) Three Way Grid semi-open shell $K n=6$

Figure 6. Displacement cloud diagram of reticulated shell.

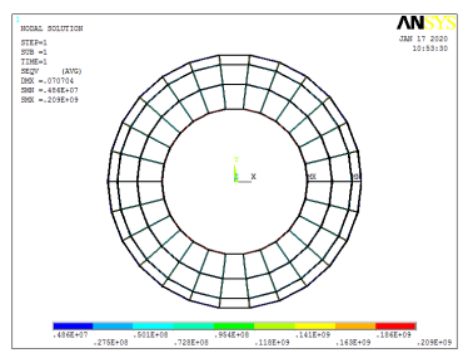

(a) Ribbed semi-open shell $K n=26$

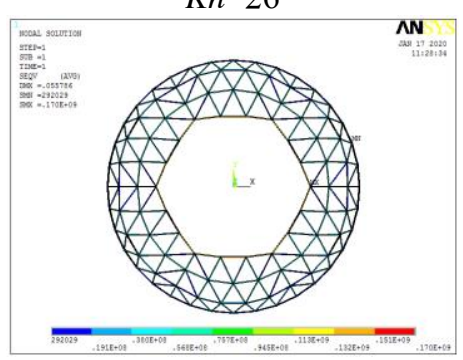

(d) Kiewit semi-open shell $K n=6$

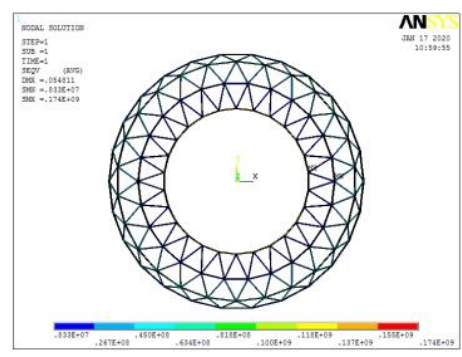

(b) Lamella semi-open shell $K n=26$

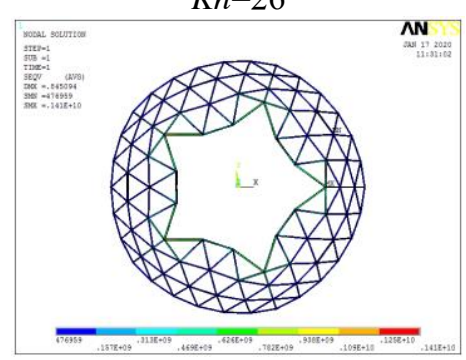

(e) Geodesic semi-open shell $K n=5$

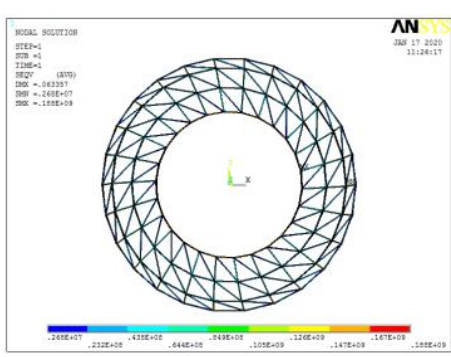

(c) Schwedler semi-open shell $K n=26$

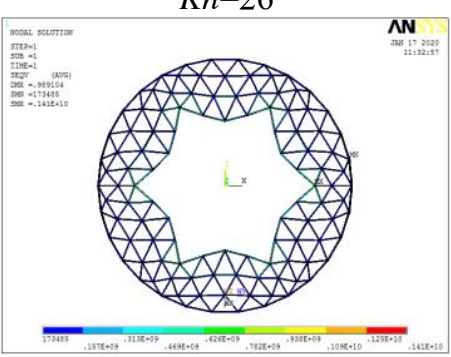

(f) Three Way Grid semi-open shell $K n=6$

Figure 7. Stress cloud diagram of reticulated shell.

Table 2. Maximum displacement and maximum stress of semi-open spherical reticulated shell.

\begin{tabular}{cccccc}
\hline types of shell & $K n$ & $\begin{array}{c}\text { maximum } \\
\text { displacement/cm }\end{array}$ & $\begin{array}{c}\text { allowable } \\
\text { displacement/cm }\end{array}$ & $\begin{array}{c}\text { maximum } \\
\text { stress/Mpa }\end{array}$ & $\begin{array}{c}\text { allowable } \\
\text { stress/Mpa }\end{array}$ \\
\hline Ribbed & 26 & 7.06 & 30 & 208.6 & 215 \\
Lamella & 26 & 5.46 & 30 & 173.6 & 215 \\
Schwedler & 26 & 6.31 & 30 & 187.7 & 215 \\
Kiewit & 6 & 5.56 & 30 & 169.9 & 215 \\
Geodesic & 5 & 83.28 & 30 & 1406.9 & 215 \\
Three Way Grid & 6 & 98.51 & 30 & 1407.2 & 215 \\
\hline
\end{tabular}

Note: the shadow data in the table has exceeded the allowable value of the structure.

The maximum displacement and maximum stress of table 3 when the latter three types of $K n$ are different. the last three reticulated shell types in table 1 are listed in

Table 3. The maximum displacement and the maximum stress of three semi-open spherical reticulated shells with different $K n$.

\begin{tabular}{cccccc}
\hline types of shell & $K n$ & $\begin{array}{c}\text { maximum } \\
\text { displacement/cm }\end{array}$ & $\begin{array}{c}\text { allowable } \\
\text { displacement/cm }\end{array}$ & $\begin{array}{c}\text { maximum } \\
\text { stress/Mpa }\end{array}$ & $\begin{array}{c}\text { allowable } \\
\text { stress/Mpa }\end{array}$ \\
\hline \multirow{3}{*}{ Ribbed } & 25 & 7.14 & 30 & 209.3 & 215 \\
& 26 & 7.06 & 30 & 208.6 & 215 \\
\hline Lamella & 27 & 6.98 & 30 & 208.0 & 215 \\
\hline
\end{tabular}




\begin{tabular}{cccccc} 
& 26 & 5.46 & 30 & 173.6 & 215 \\
& 27 & 5.39 & 30 & 173.4 & 215 \\
\hline \multirow{3}{*}{ Schwedler } & 25 & 6.39 & 30 & 189.1 & 215 \\
& 26 & 6.31 & 30 & 187.7 & 215 \\
& 27 & 6.26 & 30 & 186.6 & 215 \\
\hline
\end{tabular}

The following conclusions can be obtained from figure 6 , figure 7 , table 2 and table 3 .

(1) Under uniform distribution load, fixed hinge support is used around the structure, the maximum displacements and stresses of these six semi-open types of spherical reticulated shell structures occur in the topmost ring of the structure. The maximum displacement and stress of the Geodesic type appear at the main rib joint of the top ring. The maximum displacement and stress of the Kiewit type and the Three Way Grid type occur at the middle joint of the main rib adjacent to the top ring of the structure. The maximum displacement and stress of the other three types is evenly distributed in the top ring of the structure. The stress characteristics of the above mentioned structures should be paid attention to in engineering application.

(2) Among the six types of semi-open reticulated shells, the Kiewit type and the Lamella type have the best stress performance. Their maximum displacement and stress are less than the other four reticulated shell structures, and far less than the allowable value of the structure. Under the same conditions, the Kiewit type and the Lamella type have larger strength and stiffness reserves. The mechanical properties of the Ribbed type and the Schwedler type are close to each other. Their maximum displacement and stress of both are not beyond the allowable value of the structure. The maximum stress and displacement of the Geodesic type and the Three Way Grid type are both beyond the allowable value of the structure. They are not suitable for large-span reticulated shell structures.

\section{Conclusion}

This paper draws the following conclusions.

(1) In this paper, six methods are proposed for the joint generation and rod connection of semi-open spherical reticulated shell by using ANSYS software parametric design language APDL. These design methods are simple and efficient, which can improve the efficiency of structural design, stress analysis and the selection and optimization design of six kinds of reticulated shell in practical engineering.

(2) The modeling example shows that when the semiopen spherical reticulated shell covers the same space, the changes of the $K n$ and the reticulated shell type will cause the total number of nodes and bars to change greatly, which will lead to the great changes of the dead weight and the cost of the structure. Moreover, the internal force of the structure will be redistributed, and the mechanical performance of the reticulated shell will also change greatly.

(3) With the increase of the number of $K n$, the number of nodes and bars of reticulated shell will increase. At the same time, the dead weight and construction cost will also increase. Such change and the consequent change in the stress performance are the key cost performance factors to be analyzed in the selection and optimization design of reticulated shell.

(4) The maximum stress and displacement of the six semi-open spherical reticulated shells appear in the top ring of the structure. The maximum stress and displacement of the Geodesic type and the Three Way Grid type both exceed the allowable value of the structure. So they only apply to the small-span structure $(S \leq 80 \mathrm{~m})$. While the maximum stress and displacement of the Kiewit type and the Lamella type are far less than the allowable value of the structure. They have good mechanical performance, which can be applied to the large-span semi-open structure.

\section{Acknowledgments}

This work was financially supported by postgraduate high quality courses of Shandong Jianzhu University (YZKC201605) and projects of Shandong postgraduate innovation (SDYY08038).

\section{References}

1. Xiaoyang, L., Xiaowei Z., Shiying C. (2013) Optimum Design of Discrete Variable Shell Structure. [M]. China Architecture \& Building Press Publishing, Beijing.

2. Xiaoyang, L., Haoxin, F., Xiaowei, Z., et al. (2016) Parametric Design and Stability Analysis of Hybrid Ellipsoid Reticulated Shell. [J]. Journal of Shandong Jianzhu University., 06: 511-520.

3. Zhongjia, Z., Shaobo, L., Xiaoyang, L., et al. (2016) The Fusion of Gothic Architectural Style and Modern Reticulated Shell Structure Design: Parametric Design and Shape Optimization of Pyramid Type Folding Plate Reticulated Shell. [J]. Science Technology and Engineering., 03: 92-99.

4. Xiaoyang, L., Xiaowei, Z., Lili, H. (2012) Parametric Modeling of Six Typical Reticulated Domes. [J]. Advanced Materials Research., 424-425: 270-275.

5. Xiaoyang, L., Xiaowei, Z., Lili, H. (2012) Parametric Modeling of Five Typical Cylindrical Shells[J]. Advanced Materials Research., 424-425: 255-259.

6. Xiaoyang, L., Zhidan, W., Xiaowei, Z. (2012) Five Developed Cylinder Reticulated Shell ANSYS Parametric Modelings[J]. Applied Mechanics and Materials., 166-169: 747-750.

7. Daliang, Z., Xiaoyang, L., Shiying, C., et al. (2013) Parametric Design and Stress Characteristics of K6- 
type Triangular Pyramid System Dome. [J]. Journal of Shandong Jianzhu university., 05: 425-431.

8. JGJ 31-2003. (2003) Design Code for Sports Building. [S]. China Architecture \& Building Press Publishing, Beijing.

9. GB 50009-2016. (2016) Load Code for Building Structures. [S]. China Architecture \& Building Press Publishing, Beijing.

10. JGJ 7-2010. (2010) Technical Specification for Space Frame Structures. [S]. China Architecture \& Building Press Publishing, Beijing. 